\title{
Screening of Mungbean Accessions against Mungbean Yellow Mosaic Virus (MYMV) under Sub-tropical Irrigated Conditions of Jammu Region, India
}

\author{
Sonali Abrol* and Prachi Sharma \\ Department of Plant Pathology, Sher-e-Kashmir University of Agricultural Sciences and \\ Technology of Jammu, FOA Chatha, Jammu- 180 009, India \\ *Corresponding author
}

\begin{abstract}
A B S T R A C T
Keywords

Accessions,

Mungbean, MYMV, Resistant

Article Info

Accepted:

04 January 2018

Available Online:

10 February 2018

An investigation was carried out at Sher-e-Kashmir University of Agricultural Sciences and Technology of Jammu, during the kharif season of 2015, to assess the screening of 73 accessions of mungbean against mungbean yellow mosaic virus (MYMV). The data on morphological traits viz., days to $50 \%$ flowering, plant height $(\mathrm{cm})$, number of branches/plant, number of pods/plant, average pod weight $(\mathrm{g})$, pod length $(\mathrm{cm})$, pod diameter (mm), number of seeds/pod, 100-seed weight $(\mathrm{g})$ and days to physiological maturity were also recorded. Among different accessions, LM-250, LM-1402, M-237 and M-131 recorded significantly highest majority of traits under study and were statistically at par with each other. Whereas IPM-0102 and LM-250 were the earliest to flower and mature pods, respectively and were at par with number of accessions. On the basis of the data recorded on disease incidence, four accessions (LM-291, M-131, M-237 and LM1402) were found to be resistant, two accessions (LM-27 and LM-11686) were moderately resistant, twenty two accessions were moderately susceptible, thirty three accessions were susceptible and twelve were found to be highly susceptible to yellow mosaic disease.
\end{abstract}

\section{Introduction}

India has become self-sufficient with respect to production of cereals but still lags behind with respect to the production of pulses though the largest producer of pulses in the world with 35.7 per cent share in global production. Pulses are the main source of protein, particularly for vegetarians and contribute about 14 per cent of the total protein of an Indian average diet. Mungbean (Vigna radiata L.) is one of the most important pulse crops in India and ranks third after chickpea and pigeon pea. India ranks first in the world in area as well as production of mungbean. India is the leading producer of mungbean covering 55 per cent of total world average and 45 per cent of the total production (Rishi, 2009). Mungbean is primarily a rainy season crop but it is also suitable as a summer crop. Yellow mosaic disease (YMD) of mungbean is one of the most destructive and major constraints to productivity of grain legumes across the Indian sub-continent (Varma et al., 1992; Varma and Malathi, 2003). Yellow mosaic disease affects various 
legume crops in different proportions, which results in heavy field losses. The disease can reduce mungbean yield upto 100 per cent or even kill a plant (Kitsanachandee et al., 2013). However, there are very few reports of resistant variety/cultivar having complete resistance against yellow mosaic. For this, identification of resistant sources is a key factor. Knowledge of the mode of inheritance to MYMV in mungbean is useful for incorporation of resistance into agronomically poor, but desirable genetic resources. So far as the Jammu region is concerned, there are reports on occurrence of yellow mosaic, but the actual causal agent is not known. Thereby, keeping in view the above facts the present study was carried out with the aim of identifying the MYMV resistant progenies under sub-tropical irrigated conditions of Jammu region.

\section{Materials and Methods}

The field experiment was carried out during the kharif season of 2016 at the Research Farm of Division of Plant Pathology, Sher-eKashmir University of Agricultural Sciences and Technology of Jammu which is situated at $320-40^{\prime} \mathrm{N}$ latitude and 740-58' E longitude with an altitude of $332 \mathrm{~m}$ above mean sea level. The experiment was laid out in randomized block design with seventy three and two replications. Seventy three genotypes of mungbean (Vigna radiata) were used for yellow mosaic disease (YMD) screening studies.

The seed material was collected from different sources. Sixty nine (69) accessions of mungbean were procured from Indian Institute of Pulse Research, Kanpur (IIPR, Kanpur) and other four varieties/cultivars were procured from Pulse Research Station, Samba, local market and Department of Agriculture, Jammu (Table 1).
Resistant and susceptible sources were identified based on visual yellow mosaic symptoms on the basis of disease scale (Table 2) given by Nene et al., (1981).

The observations related to crop growth and contributing traits were recorded as per the established norms. Data obtained from the experiment were subjected to statistical analysis by following Analysis of variance technique as suggested by Panse and Sukhatme (1985).

\section{Results and Discussion}

\section{Screening for yellow mosaic under natural epiphytotics}

Of all the accessions under study, four accessions were found to be resistant, two moderately resistant, twenty two moderately susceptible, thirty three susceptible and the rest (twelve) were found to be highly susceptible under natural epiphitotic conditions (Table 3 ).

\section{Growth and yield parameters}

The data showed variation among different accessions. Mungbean accessions were also evaluated for various growth and yield parameters viz., days to 50 percent flowering, plant height, number of branches/plant, number of pods/plant, average pod weight, pod length, pod diameter, number of seeds/pod, 100-seed weight and days to physiological maturity to determine the effect of yellow mosaic disease in different accessions (Table 4 and 5). Of all the accessions, M-237, LM-1402, M-131 and LM291 recorded maximum values for majority of the traits under study. However, these accessions took longer time for days to 50 percent flowering and thus also took more days to physiological maturity. 
Table.1 List of mungbean accessions along with source of procurement

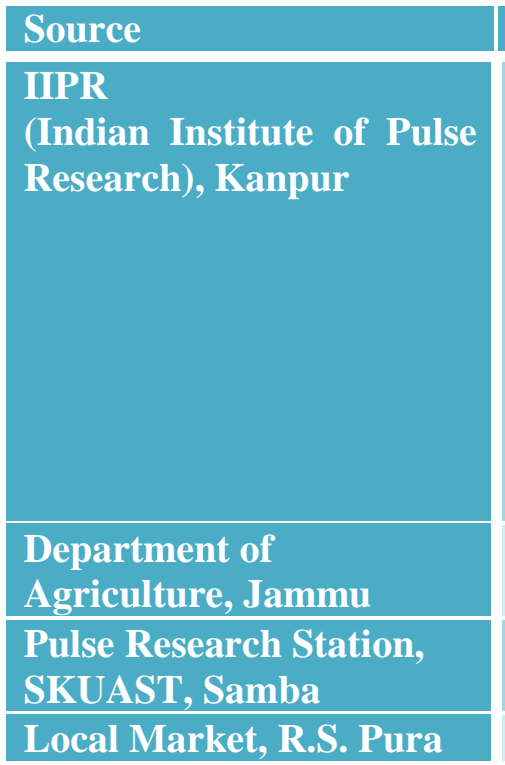

\section{Accession Name}

LM-12, LM-197, LM-250, LM-1494, IM-636, LM-302, LM-309, LM328, LM-1021, LM-1, LM-257, LM-344, LM-13487, LM-220, LM1397, LM-285,LM-27, LM-11686, LM-253, LM-274, LM-1485, LM316, LM-34, L-4616, LM-150, LM-228, LM-1169, LM-241, LM-194, LM-1598, LM-19, LM-1029, LL-1119, LM-104, LM-1510, LM-101, LM-291, IC-8839, LM-226, LAM-66127, LM-294, LM-3, IM-250, LM258, LM-232, LM-23, IM-259, LM-249, LM-1193, LM-1678, LM1564, LM-16, LM-3, LM-1089, M-18, LM-303, LM-326, M-131,INN644, M-237, LM-312, LM-1402, LM-174, LM-236, LM-1561, INBN208, LM-159, LM-137

SML-668, IPM-0102

\section{MOONG-ML}

\section{R.S PURA LOCAL}

Table.2 Disease grade scale for yellow mosaic

\begin{tabular}{|c|c|}
\hline Rating & Description \\
\hline $\mathbf{0}$ & No symptoms on any plant \\
\hline 1 & Yellow mottle or necrotic mottle in upto $1 \%$ plants \\
\hline 3 & Yellow mottle or necrotic mottle in traces on $1.1-10 \%$ plants \\
\hline 5 & $\begin{array}{l}\text { Necrotic mottle/ mild mottle/ mild symptoms; } 10.1-25 \% \text { plants; No reduction in } \\
\text { plant growth; No yield loss }\end{array}$ \\
\hline 7 & $\begin{array}{l}\text { Yellow mottle symptoms not covering the whole leaf lamina on } 25.1-50 \% \text { plants; } \\
\text { reduction in leaf and plant growth }\end{array}$ \\
\hline 9 & $\begin{array}{l}\text { Yellow mottle symptoms on }>50 \% \text { plants; severe reduction in leaf and plant growth } \\
\text { as well as pod formation and death of plant. }\end{array}$ \\
\hline
\end{tabular}

Table.3 Diversity in symptoms in mungbean accessions infected with yellow mosaic virus under natural epiphytotics

\begin{tabular}{|l|l|l|c|c|}
\hline S. No. & Accession Name & Symptoms & Disease Grade & Reaction* \\
\hline 1. & LM-12 & Severe Yellow mosaic & 9 & HS \\
\hline 2. & LM-197 & Yellow spots & 7 & MS \\
\hline 3. & LM-250 & Severe Yellow mosaic & 9 & HS \\
\hline 4. & LM-1494 & Mild Flecks & 5 & S \\
\hline 5. & IM-636 & Yellow Flecks & 9 & HS \\
\hline 6. & LM-302 & Marginal Yellow mosaic & 5 & S \\
\hline 7. & LM-309 & Mild Yellow mosaic & 7 & MS \\
\hline 8. & LM-328 & Severe Yellow mosaic & 9 & HS \\
\hline 9. & LM-1021 & Yellow flecks & 7 & MS \\
\hline
\end{tabular}




\begin{tabular}{|c|c|c|c|c|}
\hline 10. & LM-1 & Marginal yellow mosaic & 5 & $\mathrm{~S}$ \\
\hline 11. & LM-257 & Marginal yellow mosaic & 5 & $\mathrm{~S}$ \\
\hline 12. & LM-344 & Severe Yellow mosaic & 7 & MS \\
\hline 13. & LM-13487 & Mild Yellow flecks & 5 & $\mathrm{~S}$ \\
\hline 14. & LM-220 & Marginal yellow mosaic & 9 & HS \\
\hline 15. & LM-1397 & Yellow mosaic & 7 & MS \\
\hline 16. & LM-285 & Marginal yellow mosaic & 5 & $\mathrm{~S}$ \\
\hline 17. & LM-27 & Yellow mosaic & 3 & MR \\
\hline 18. & LM-11686 & Yellow flecks & 3 & MR \\
\hline 19. & LM-253 & Marginal yellow mosaic & 7 & MS \\
\hline 20. & LM-274 & Severe yellow mosaic & 7 & MS \\
\hline 21. & LM-1485 & Mild yellow flecks & 5 & $\mathrm{~S}$ \\
\hline 22. & LM-316 & Marginal yellow mosaic & 5 & $\mathrm{~S}$ \\
\hline 23. & LM-34 & Yellow spots & 7 & MS \\
\hline 24. & L-4616 & Yellow flecks & 5 & $\mathrm{~S}$ \\
\hline 25. & LM-150 & Yellow flecks & 5 & $\mathrm{~S}$ \\
\hline 26. & LM-228 & Yellow mosaic & 7 & MS \\
\hline 27. & LM-1169 & Yellow mosaic & 7 & MS \\
\hline 28. & LM-241 & Yellow mosaic & 7 & MS \\
\hline 29. & LM-194 & Yellow spots & 7 & MS \\
\hline 30. & LM-1598 & Yellow spots & 7 & MS \\
\hline 31. & LM-19 & Marginal yellow mosaic & 7 & MS \\
\hline 32. & LM-1029 & Yellow spots & 7 & MS \\
\hline 33. & LL-1119 & Yellow spots & 5 & $\mathrm{~S}$ \\
\hline 34. & LM-104 & Marginal yellow mosaic & 5 & $\mathrm{~S}$ \\
\hline 35. & LM-1510 & Yellow flecks & 7 & MS \\
\hline 36. & LM-1560 & Yellow spots & 5 & $\mathrm{~S}$ \\
\hline 37. & LM-101 & Mild yellow mosaic & 5 & $\mathrm{~S}$ \\
\hline 38. & LM-291 & No disease & 0 & $\mathrm{R}$ \\
\hline 39. & IC- 8839 & Yellow mosaic & 5 & $\mathrm{~S}$ \\
\hline 40. & LM-226 & Yellow mosaic & 5 & $\mathrm{~S}$ \\
\hline 41. & LAM-66127 & Yellow mosaic & 5 & $\mathrm{~S}$ \\
\hline 42. & LM-294 & Yellow flecks & 7 & MS \\
\hline 43. & LM-3 & Marginal yellow mosaic & 5 & $\mathrm{~S}$ \\
\hline 44. & IM-250 & Yellow flecks & 5 & $\mathrm{~S}$ \\
\hline 45. & LM-258 & Yellow flecks & 5 & $\mathrm{~S}$ \\
\hline 46. & LM-232 & Yellow mosaic & 7 & MS \\
\hline 47. & LM-23 & Yellow flecks & 5 & $\mathrm{~S}$ \\
\hline 48. & IM-259 & Yellow flecks & 7 & MS \\
\hline 49. & LM-249 & Yellow flecks & 5 & $\mathrm{~S}$ \\
\hline 50. & LM-1193 & Yellow flecks & 5 & $\mathrm{~S}$ \\
\hline 51. & LM-1678 & Marginal yellow mosaic & 5 & $\mathrm{~S}$ \\
\hline 52. & LM-1564 & Severe yellow mosaic & 9 & HS \\
\hline 53. & LM-16 & Yellow mosaic & 5 & $\mathrm{~S}$ \\
\hline 54. & LM-3 & Severe yellow mosaic & 9 & HS \\
\hline 55. & LM-1089 & Severe yellow mosaic & 9 & HS \\
\hline 56. & M-18 & Yellow spots & 9 & HS \\
\hline
\end{tabular}




\begin{tabular}{|c|c|c|c|c|}
\hline 57. & LM-303 & Yellow flecks & 5 & $\mathrm{~S}$ \\
\hline 58. & LM-326 & Marginal yellow mosaic & 7 & MS \\
\hline 59. & M-131 & No disease & 0 & $\mathrm{R}$ \\
\hline 60. & INN-646 & Marginal yellow mosaic & 5 & S \\
\hline 61. & M-237 & No disease & 0 & $\mathrm{R}$ \\
\hline 62. & LM-312 & Yellow mosaic & 5 & S \\
\hline 63. & LM-1402 & No disease & 0 & $\mathrm{R}$ \\
\hline 64. & LM-174 & Yellow mosaic & 9 & HS \\
\hline 65. & LM-236 & Yellow flecks & 5 & $\mathrm{~S}$ \\
\hline 66. & LM-1561 & Yellow spots & 9 & HS \\
\hline 67. & INBN-208 & Marginal Yellow mosaic & 5 & $\mathrm{~S}$ \\
\hline 68. & LM-159 & Yellow spots & 7 & MS \\
\hline 69. & LM-137 & Yellow spots & 7 & MS \\
\hline 70. & SML-668 & Yellow mosaic & 5 & $\mathrm{~S}$ \\
\hline 71. & R.S PURA LOCAL & Severe yellow mosaic & 9 & HS \\
\hline 72. & IPM-01-02 & Yellow flecks & 5 & $\mathrm{~S}$ \\
\hline 73. & Moong ML-1 & Yellow flecks & 5 & S \\
\hline
\end{tabular}

Note $*$ HS $=$ Highly susceptible, MS =Moderately susceptible, $\mathrm{S}=$ Susceptible, $\mathrm{R}=$ Resistant, MR= Moderately resistant

Table.4 Evaluation of growth and yield parameters in mungbean accessions under field conditions during kharif 2015

\begin{tabular}{|l|c|c|c|c|c|}
\hline $\begin{array}{l}\text { Accession } \\
\text { name }\end{array}$ & $\begin{array}{c}\text { Plant height } \\
(\mathbf{c m})\end{array}$ & $\begin{array}{c}\text { Days to 50\% } \\
\text { flowering }\end{array}$ & $\begin{array}{c}\text { No. of } \\
\text { branches/plant }\end{array}$ & $\begin{array}{c}\text { No. of } \\
\text { pods/plant }\end{array}$ & $\begin{array}{c}\text { Avg. Pod } \\
\text { weight }(\mathbf{g})\end{array}$ \\
\hline LM-12 & 70.96 & 34.50 & 10.50 & 10.70 & 0.29 \\
\hline LM-197 & 72.46 & 34.17 & 9.50 & 10.75 & 0.28 \\
\hline LM-250 & 53.84 & 33.99 & 8.50 & 11.80 & 0.31 \\
\hline LM-1494 & 63.63 & 35.00 & 10.25 & 10.85 & 0.28 \\
\hline IM-636 & 47.38 & 33.91 & 11.92 & 11.10 & 0.27 \\
\hline LM-302 & 63.04 & 35.11 & 12.42 & 11.00 & 0.32 \\
\hline LM-309 & 56.29 & 33.11 & 10.50 & 8.85 & 0.30 \\
\hline LM-328 & 53.88 & 34.17 & 10.84 & 11.85 & 0.31 \\
\hline LM-1021 & 60.88 & 34.17 & 10.92 & 10.05 & 0.27 \\
\hline LM-1 & 64.13 & 33.00 & 11.59 & 12.80 & 0.28 \\
\hline LM-257 & 56.88 & 34.18 & 11.00 & 9.25 & 0.28 \\
\hline LM-344 & 55.79 & 35.67 & 12.00 & 10.70 & 0.33 \\
\hline LM-13487 & 69.80 & 34.17 & 11.84 & 9.59 & 0.31 \\
\hline LM-220 & 54.05 & 34.69 & 9.75 & 10.70 & 0.32 \\
\hline LM-1397 & 47.48 & 36.50 & 11.84 & 10.50 & 0.28 \\
\hline LM-285 & 57.30 & 36.16 & 10.75 & 10.85 & 0.28 \\
\hline LM-27 & 70.71 & 34.00 & 11.75 & 11.70 & 0.32 \\
\hline LM-11686 & 62.96 & 35.37 & 11.17 & 11.42 & 0.31 \\
\hline LM-253 & 65.38 & 38.50 & 12.33 & 11.05 & 0.29 \\
\hline
\end{tabular}




\begin{tabular}{|l|l|l|l|l|l|}
\hline LM-274 & 50.80 & 36.71 & 11.50 & 10.09 & 0.32 \\
\hline LM-1485 & 51.96 & 34.61 & 11.34 & 10.92 & 0.29 \\
\hline LM-316 & 59.80 & 34.62 & 12.75 & 9.67 & 0.28 \\
\hline LM-34 & 60.30 & 34.81 & 11.00 & 10.09 & 0.28 \\
\hline LM-4616 & 48.52 & 34.50 & 11.92 & 11.47 & 0.33 \\
\hline LM-150 & 57.21 & 33.50 & 12.33 & 10.17 & 0.31 \\
\hline LM-228 & 64.96 & 33.67 & 12.00 & 11.05 & 0.30 \\
\hline LM-1169 & 47.62 & 34.61 & 11.83 & 11.06 & 0.31 \\
\hline LM-241 & 43.38 & 34.67 & 10.99 & 10.86 & 0.30 \\
\hline LM-194 & 63.63 & 36.17 & 12.25 & 11.50 & 0.32 \\
\hline LM-1598 & 70.88 & 36.83 & 11.58 & 11.20 & 0.32 \\
\hline LM-19 & 57.80 & 36.46 & 11.42 & 11.80 & 0.28 \\
\hline LM-1029 & 76.96 & 34.17 & 9.92 & 11.00 & 0.29 \\
\hline LL-1119 & 43.38 & 35.12 & 11.58 & 10.50 & 0.31 \\
\hline LM-104 & 47.46 & 37.50 & 10.59 & 13.05 & 0.28 \\
\hline LM-1510 & 58.13 & 35.50 & 11.00 & 11.80 & 0.29 \\
\hline LM-1560 & 45.04 & 35.11 & 10.75 & 10.64 & 0.29 \\
\hline LM-101 & 50.80 & 36.12 & 10.67 & 11.56 & 0.33 \\
\hline LM-291 & $\mathbf{8 2 . 4 2}$ & $\mathbf{3 9 . 5 0}$ & $\mathbf{1 4 . 5 0}$ & $\mathbf{1 4 . 8 4}$ & $\mathbf{0 . 4 1}$ \\
\hline IC-8839 & 45.84 & 34.17 & 11.67 & 10.90 & 0.31 \\
\hline LM-226 & 42.50 & 35.17 & 11.62 & 11.65 & 0.32 \\
\hline LAM-66127 & 44.14 & 34.00 & 11.59 & 10.90 & 0.30 \\
\hline LM-294 & 45.63 & 36.17 & 12.09 & 11.85 & 0.30 \\
\hline LM-3 & 51.75 & 33.50 & 11.50 & 11.35 & 0.28 \\
\hline LM-250 & 48.24 & 34.64 & 11.84 & 10.80 & 0.29 \\
\hline LM-258 & 54.33 & 33.50 & 12.59 & 12.20 & 0.28 \\
\hline LM-232 & 52.33 & 33.78 & 10.25 & 14.46 & 0.28 \\
\hline LM-23 & 44.02 & 35.89 & 11.75 & 12.10 & 0.28 \\
\hline IM-259 & 46.75 & 34.83 & 10.82 & 11.50 & 0.29 \\
\hline LM-249 & 46.85 & 36.00 & 9.92 & 10.90 & 0.31 \\
\hline LM-1193 & 44.11 & 35.00 & 11.25 & 11.50 & 0.31 \\
\hline LM-1678 & 48.88 & 34.00 & 11.00 & 11.20 & 0.28 \\
\hline LM-1564 & 43.09 & 34.00 & 9.84 & 11.00 & 0.32 \\
\hline LM-16 & 48.80 & 35.50 & 11.17 & 12.65 & 0.29 \\
\hline LM-3 & 42.00 & 33.00 & 11.39 & 11.95 & 0.28 \\
\hline LM-1089 & 47.42 & 34.12 & 11.84 & 11.70 & 0.28 \\
\hline M-18 & 49.52 & 34.50 & 11.83 & 11.15 & 0.29 \\
\hline LM-303 & 46.62 & 34.69 & 11.67 & 12.10 & 0.26 \\
\hline LM-326 & 50.83 & 34.61 & 11.42 & 9.50 & 0.31 \\
\hline M-131 & $\mathbf{8 0 . 8 4}$ & $\mathbf{3 9 . 5 3}$ & $\mathbf{1 4 . 6 7}$ & $\mathbf{1 5 . 5 0}$ & $\mathbf{0 . 3 8}$ \\
\hline & & & & & \\
\hline
\end{tabular}




\begin{tabular}{|l|l|l|l|c|c|}
\hline INN-646 & 49.67 & 36.50 & 9.25 & 8.67 & 0.28 \\
\hline M-237 & $\mathbf{7 9 . 4 2}$ & $\mathbf{4 0 . 5 0}$ & $\mathbf{1 4 . 0 0}$ & $\mathbf{1 4 . 5 0}$ & $\mathbf{0 . 3 9}$ \\
\hline LM-312 & 40.58 & 34.00 & 11.42 & 10.55 & 0.32 \\
\hline LM-1402 & $\mathbf{8 1 . 2 4}$ & $\mathbf{4 0 . 0 0}$ & $\mathbf{1 4 . 4 2}$ & $\mathbf{1 4 . 6 7}$ & $\mathbf{0 . 4 0}$ \\
\hline LM-174 & 43.39 & 34.00 & 9.75 & 9.75 & 0.28 \\
\hline LM-236 & 42.14 & 33.50 & 10.50 & 11.00 & 0.28 \\
\hline LM-1561 & 44.64 & 34.17 & 11.25 & 11.45 & 0.30 \\
\hline INBN-208 & 45.39 & 34.72 & 10.20 & 11.50 & 0.32 \\
\hline LM-159 & 41.14 & 33.50 & 11.64 & 11.20 & 0.32 \\
\hline LM-137 & 41.97 & 35.12 & 10.47 & 12.10 & 0.29 \\
\hline SML-668 & 59.09 & 35.17 & 10.39 & 12.10 & 0.30 \\
\hline R.S Pura & 60.71 & 34.66 & 12.32 & 10.09 & 0.25 \\
\hline Local & & & & & \\
\hline IPM-01 & 45.57 & $\mathbf{3 3 . 0 0}$ & 11.75 & 9.97 & 0.28 \\
\hline MOONG- & 71.14 & 34.00 & 12.95 & 10.67 & 0.29 \\
\hline MIL & & & & & \\
\hline SEm ( \pm & 2.02 & 1.26 & 0.80 & 0.49 & 0.02 \\
\hline CD $(\mathbf{P}=0.05)$ & 5.68 & 3.54 & 2.25 & 1.38 & 0.06 \\
\hline
\end{tabular}

Table.5 Evaluation of yield parameters in mungbean accessions under field conditions during kharif 2015

\begin{tabular}{|l|c|c|c|c|c|}
\hline Accession name & $\begin{array}{c}\text { Pod length } \\
(\mathbf{c m})\end{array}$ & $\begin{array}{c}\text { Pod } \\
\text { diameter } \\
(\mathbf{m m})\end{array}$ & $\begin{array}{c}\text { No. of } \\
\text { seeds/pod }\end{array}$ & $\begin{array}{c}\text { 100-seed } \\
\text { weight (gm) }\end{array}$ & $\begin{array}{c}\text { Days to } \\
\text { physiological } \\
\text { maturity }\end{array}$ \\
\hline LM-12 & 5.72 & 5.80 & 7.35 & 2.92 & 65.62 \\
\hline LM-197 & 6.15 & 6.05 & 8.35 & 2.89 & 67.06 \\
\hline LM-250 & 6.60 & 6.20 & 8.80 & 2.88 & 62.56 \\
\hline LM-1494 & 6.10 & 5.75 & 8.30 & 3.16 & 67.56 \\
\hline IM-636 & 6.38 & 5.90 & 8.55 & 3.43 & 70.14 \\
\hline LM-302 & 7.01 & 6.00 & 9.15 & 3.26 & 66.18 \\
\hline LM-309 & 6.63 & 5.95 & 8.80 & 3.55 & 68.65 \\
\hline LM-328 & 7.15 & 5.90 & 9.45 & 3.18 & 69.50 \\
\hline LM-1021 & 7.13 & 6.05 & 9.30 & 3.65 & 66.73 \\
\hline LM-1 & 7.19 & 5.75 & 9.35 & 2.95 & 65.61 \\
\hline LM-257 & 6.90 & 6.10 & 9.10 & 3.50 & 67.13 \\
\hline LM-344 & 7.29 & 6.15 & 9.45 & 3.41 & 69.79 \\
\hline LM-13487 & 6.71 & 5.75 & 8.80 & 3.55 & 70.63 \\
\hline LM-220 & 7.18 & 5.80 & 9.40 & 3.71 & 70.51 \\
\hline LM-1397 & 6.16 & 6.05 & 8.37 & 3.65 & 66.45 \\
\hline LM-285 & 7.10 & 5.90 & 9.35 & 3.41 & 66.41 \\
\hline LM-27 & 5.92 & 6.20 & 6.70 & 3.45 & 65.89 \\
\hline
\end{tabular}




\begin{tabular}{|c|c|c|c|c|c|}
\hline LM-11686 & 7.00 & 6.10 & 9.15 & 3.32 & 65.50 \\
\hline LM-253 & 6.70 & 6.30 & 8.90 & 3.85 & 70.62 \\
\hline LM-274 & 6.00 & 6.00 & 8.15 & 3.65 & 69.99 \\
\hline LM-1485 & 6.60 & 5.95 & 8.80 & 3.55 & 68.57 \\
\hline LM-316 & 7.05 & 6.00 & 9.25 & 3.45 & 65.26 \\
\hline LM-34 & 6.96 & 6.05 & 9.10 & 3.55 & 65.30 \\
\hline LM-4616 & 7.02 & 5.75 & 9.25 & 3.40 & 66.30 \\
\hline LM-150 & 6.50 & 5.65 & 8.75 & 3.65 & 66.73 \\
\hline LM-228 & 7.03 & 5.80 & 9.25 & 3.80 & 69.56 \\
\hline LM-1169 & 7.14 & 5.90 & 9.35 & 3.65 & 70.50 \\
\hline LM-241 & 6.85 & 6.20 & 8.95 & 3.71 & 65.76 \\
\hline LM-194 & 6.71 & 6.05 & 8.95 & 3.30 & 65.63 \\
\hline LM-1598 & 6.91 & 6.25 & 9.15 & 3.00 & 69.72 \\
\hline LM-19 & 6.80 & 6.25 & 9.00 & 3.50 & 66.21 \\
\hline LM-1029 & 6.88 & 6.10 & 9.40 & 3.60 & 70.67 \\
\hline LL-1119 & 7.20 & 5.75 & 9.45 & 3.46 & 70.55 \\
\hline LM-104 & 6.92 & 5.65 & 9.15 & 3.38 & 66.43 \\
\hline LM-1510 & 6.82 & 5.70 & 9.05 & 3.28 & 67.88 \\
\hline LM-1560 & 6.89 & 6.00 & 9.15 & 3.24 & 67.62 \\
\hline LM-101 & 6.34 & 5.80 & 8.55 & 3.45 & 67.08 \\
\hline LM-291 & 7.95 & 6.00 & 9.90 & 4.18 & 80.45 \\
\hline IC-8839 & 6.90 & 5.65 & 9.05 & 3.40 & 70.18 \\
\hline LM-226 & 7.08 & 5.75 & 9.45 & 3.60 & 65.78 \\
\hline LAM-66127 & 6.90 & 6.25 & 9.05 & 3.60 & 70.08 \\
\hline LM-294 & 7.03 & 6.00 & 9.20 & 3.26 & 69.99 \\
\hline LM-3 & 6.79 & 6.00 & 9.10 & 3.40 & 65.87 \\
\hline LM-250 & 6.89 & 6.05 & 9.45 & 3.22 & 66.56 \\
\hline LM-258 & 6.74 & 6.10 & 8.80 & 3.51 & 66.64 \\
\hline LM-232 & 7.08 & 6.20 & 9.35 & 3.30 & 67.18 \\
\hline LM-23 & 6.92 & 6.00 & 9.00 & 3.26 & 66.80 \\
\hline IM-259 & 7.31 & 5.70 & 9.40 & 3.53 & 66.56 \\
\hline LM-249 & 6.77 & 5.85 & 8.95 & 3.60 & 70.06 \\
\hline LM-1193 & 6.67 & 5.65 & 8.88 & 3.40 & 70.34 \\
\hline LM-1678 & 6.49 & 6.20 & 8.75 & 3.46 & 66.39 \\
\hline LM-1564 & 7.15 & 6.10 & 9.45 & 3.59 & 68.01 \\
\hline LM-16 & 7.04 & 5.85 & 9.25 & 3.67 & 66.42 \\
\hline LM-3 & 7.04 & 6.05 & 9.25 & 3.45 & 70.14 \\
\hline LM-1089 & 6.53 & 6.00 & 9.20 & 3.35 & 66.27 \\
\hline M-18 & 6.99 & 5.95 & 9.20 & 3.36 & 66.14 \\
\hline LM-303 & 7.02 & 5.70 & 9.25 & 3.47 & 67.68 \\
\hline LM-326 & 6.85 & 5.90 & 9.15 & 3.22 & 67.05 \\
\hline M-131 & 8.00 & 6.00 & 10.25 & 4.05 & 79.56 \\
\hline INN-646 & 6.12 & 6.15 & 8.35 & 3.59 & 69.61 \\
\hline
\end{tabular}




\begin{tabular}{|c|c|c|c|c|c|}
\hline M-237 & 8.07 & 6.25 & 10.30 & 4.15 & 80.27 \\
\hline LM-312 & 7.02 & 6.25 & 9.25 & 3.70 & 70.01 \\
\hline LM-1402 & 8.14 & 6.05 & 10.10 & 4.06 & 80.43 \\
\hline LM-174 & 6.50 & 6.00 & 8.70 & 3.40 & 70.13 \\
\hline LM-236 & 6.61 & 5.85 & 8.75 & 3.50 & 66.54 \\
\hline LM-1561 & 6.91 & 6.05 & 9.40 & 3.30 & 66.52 \\
\hline INBN-208 & 7.11 & 5.80 & 9.30 & 3.55 & 65.56 \\
\hline LM-159 & 6.58 & 6.15 & 8.75 & 3.30 & 69.62 \\
\hline LM-137 & 6.77 & 6.00 & 9.25 & 3.20 & 70.01 \\
\hline SML-668 & 6.95 & 6.10 & 9.65 & 3.20 & 70.01 \\
\hline R.S PURA LOCAL & 4.37 & 6.15 & 5.60 & 3.25 & 65.56 \\
\hline IPM-01 & 6.85 & 6.00 & 9.55 & 3.50 & 70.28 \\
\hline MOONG-ML & 7.03 & 6.10 & 9.65 & 3.46 & 70.33 \\
\hline $\operatorname{SEm}( \pm)$ & 0.27 & 0.18 & 0.41 & 0.13 & 2.45 \\
\hline $\mathrm{CD}(\mathrm{P}=0.05)$ & 0.76 & NS & 1.15 & 0.36 & 6.90 \\
\hline
\end{tabular}

The accessions viz. M-237, LM-1402, M-131 and LM-291 recording significantly higher number of pods/plant, average pod weight, pod length, number of seeds/pod and 100-seed weight may be attributed to the resistance of these accessions to yellow mosaic virus. In the present set of germplasm significant level of diversity in terms of various growth and yield characters was observed, which is well evident from the significant values in all the traits studied except for pod diameter in different accessions.

\section{References}

Kitsanachandee, R., Somta, P., Chatchawankanphanich, O., Akhtar, K. P., Shah, T. M., Nair, R., Bains, M., Sirari, T.S., Kaur, A. and Srinives, P. 2013. Detection of quantitative trait loci for mungbean yellow mosaic India virus (MYMIV) resistance in mungbean (Vigna radiata (L.) Wilczek) in India and Pakistan. Breed. Sci., 63: 367-373.
Nene, Y.L., Haware, M.P. and Reddy, M.V. 1981. Chickpea diseases: resistancescreening techniques. International Crops Research Institute for the Semi-Arid Tropics, 10: 9-10.

Panse, V.G. and Sukhatme, P.V. 1985. Statistical Methods for Agricultural Workers, $4^{\text {th }}$ enlarged edition, Indian Council of Agricultural Research, New Delhi.

Rishi, A., Narayan, K. and Quot, R. 2009. Significant plant virus diseases in India and a glimpse of modern disease management technology. J. Gen. Plant Pathol., 75: 1-18.

Varma A., Dhar, A.K., Mandal, B. 1992. MYMV transmission and control in India. Asian Vegetable Research and Development Centre: 8-27.

Varma, A. and Malathi, V. G. 2003. Emerging geminivirus problems: A serious threat to crop production. Ann. Appl. Biol., 14: 145-164.

\section{How to cite this article:}

Sonali Abrol and Prachi Sharma. 2018. Screening of Mungbean Accessions against Mungbean Yellow Mosaic Virus (MYMV) under Sub-tropical Irrigated Conditions of Jammu Region, India. Int.J.Curr.Microbiol.App.Sci. 7(02): 42-50. doi: https://doi.org/10.20546/ijcmas.2018.702.007 